\title{
Divide and conquer
}

\section{A key element of performing good cell-biology experiments is starting with exactly the right cells. Michael Eisenstein takes a look at the technologies that can make this possible.}

What do you get when you cross an ink-jet printer with a Coulter counter? It's not a riddle; scientists at Los Alamos National Laboratory in New Mexico asked the question 40 years ago, and the answer turned out to be the cell sorter.

Los Alamos researcher Mack Fulwyler created a prototype in 1965, which used vibration to generate tiny droplets from a jet of solution containing red blood cells; the individual cells in each droplet could then be subjected to rapid volumetric analysis and sorting. Fulwyler's prototype came to the attention of Stanford University researcher Leonard Herzenberg. "I was working on immunofluorescence in immunology and genetics," he says, "and had realized that there was a need for the means to sort cells according to the molecules they display on their surface."

Herzenberg and his colleagues adapted Fulwyler's design to produce an instrument that could sort cells depending on the presence or absence of molecules identified by fluorescent labels - the first example of fluorescenceactivated cell sorting (FACS). Today, FACS has not only aged gracefully but is arguably in its prime, embraced by nearly everybody looking

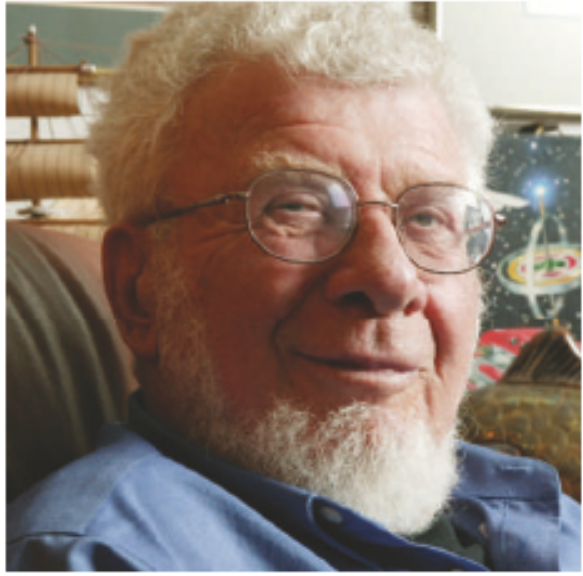

Leonard Her zenber g devised early cell sorters.

to pluck specific cells out of complex mixtures. Herzenberg's patent was licensed by Becton Dickinson (BD), which developed the first commercial instrument and currently holds the trademark for the term 'FACS. Early FACS fans include Dutch researcher Gerrit Van den Engh, whose refinements enhanced sorting rates. "I designed a different, digital parallel post-processing scheme," says Van den Engh. "ू "By digitizing the signal as quickly as possible, we could take the cells in parallel and we could put an error-tracking code on the information, so that we could check whether events were being dealt with properly."

Cytomation, later acquired by Dako in Glostrup, Denmark, made Van den Engh's patent the foundation for MoFlo, the firsthigh-speed commercial sorter. MoFlo pushes the speed envelope, sorting up to 70,000 objects per second - although researchers often use lower rates to optimize recovery and sort accuracy. Dako has also ensured that new expansions and components are suitable for use both with current and older systems. "Our modern upgrades are reverse-compatible, even with MoFlo machines from eight or nine years ago," says Cytomation's founder, George Malachowski.

Meanwhile BD Biosciences, a segment of BD in San Jose, California, recently introduced its most advanced cell sorter to date. The BD FACSAria benefits from its small size, being one of the few bench-top systems on the market, and from an alternative approach to presorting analysis. Most sorters use the 'jet-in-air'

\section{THE GENTLE TOUCH}

Sorting individual cells is not a problem, but what if a researcher wants to sort clusters of cells, or even whole embryos?

Union Biometrica of Holliston, Massachusetts, offers a potential solution in the shape of its COPAS family of instruments. These, it says, can work with objects ranging from pancreatic islets up to zebrafish hatchlings. Theprinciple is similar to fluorescence-activated cellsorting (FACS) - objects in solution pass through a specially designed flow-cell, where they are op tically profiled according to as many as fivedifferent sorting parameters (which can include threefluorescent waveleng ths), and specimens of interest are diverted and collected.

But some design adjustments werenecessary to protect the integrity of the multicellular objects being sorted. For one thing, rather than using charge-based sorting of droplets, COPAS uses rapid puffis of air to gently divert 'slices' from the flow stream that contain objects of interest. COPAS also uses lower flowrates than FACS, sorting between

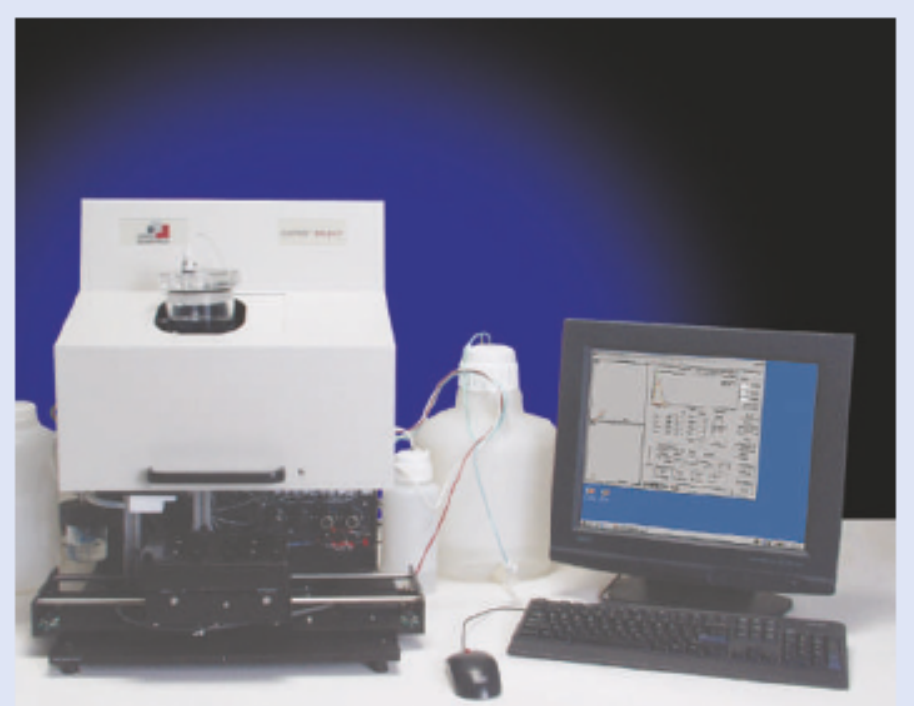

Union Biometrica's BioSorter COPAS can sort clusters of cells.

100 and 300 objects per second. "Wes acrifice speed for gentle flow, which results in increased viability and less des truction of what we're analysing," says Rock
Pulak, Union Biometrica's director of life sciences. "It's still much faster than trying to analyse these samenumbers of cell clusters by microscope."

In addition, the lower speeds mean that it is possible to do limited analysis of fluorescence localization within the objects being sorted. Pulak speculates that future instruments may even be able to perform actual imaging during the sorting process.

Sometimes even singlecells prefer a lighter touch, and an ongoing collaboration between the company and the Jos lin Diabetes Center in Boston has shown that COPAS is also us eful for sorting adipocytes and hepatocytes.

"These are larger cells that are verydelicate and subject to sensitivities with respect to shear force," says Pulak. "We are finding that our technology is appropriate for these kinds of applications." 